\title{
Minimal Absorption Measurements
}

\author{
Serge Massar $^{1}$, Graeme Mitchison $^{2}$ and Stefano Pironio ${ }^{1}$ \\ ${ }^{1}$ Service de Physique Théorique, Université Libre de Bruxelles, CP 225, Bvd. du Triomphe, B1050 Bruxelles, Belgium. \\ ${ }^{2}$ MRC Laboratory of Molecular Biology, Hills Road, Cambridge CB2 2QH, UK.
}

(February 22, 2001)

\begin{abstract}
In this paper we consider the problem of trying to make an image of an object while minimizing the number of photons absorbed by the object. We call protocols which achieve this goal "minimal absorption measurements". Such imaging techniques are particularly relevant in situations where the object can be damaged by the radiation used to make the image. Our main results are bounds that relate the minimum number of absorbed photons to the sensitivity of the measurement. In the case where the object consists of a single pixel, we show that these bounds can be approached either by simply counting the number of photons absorbed by the object or by a simple interferometric setup, depending on the details of the problem. In the case where the object consists of many pixels, we give an example where our bound can be approached when all the pixels are addressed collectively, whereas addressing each pixel individually implies an increase in the number of absorbed photons by a factor logarithmic in the number of pixels. Finally, we consider some special situations where our bound does not apply, and where interferometric methods can make large gains.
\end{abstract}

PACS numbers: $03.65 . \mathrm{Bz}$

\section{INTRODUCTION}

We examine the possible advantages of using the quantum properties of light for making images of photosensitive objects, i.e. objects that can be damaged by the photons that are used to make the image. The recent advances in quantum information processing suggest that using the quantum nature of light may provide advantages over classical imaging techniques. In particular Elitzur and Vaidman [1] have proposed "interaction-free measurements" (generalized in 2]) that can determine whether a completely absorbing object is present or absent with infinitesimal probability that the object absorbs a photon. Absorption-free measurements (as we prefer to call them, rather than "interaction-free", since our terminology reduces the number of inverted commas) have been further generalized in [3] to the case where the object is semi-transparent. And in [4] it was shown that an interferometric setup is useful when one wants to determine simultaneously the probability that a semitransparent photosensitive object absorbs a photon and the phase it induces on a photon that traverses it but is not absorbed (i.e. one wants to measure both the real and imaginary part of the transparency $\alpha$ introduced below).

Thus quantum mechanics may provide a powerful new way of making images of photosensitive objects, and this has motivated various experimental implementations of absorption-free measurements 2. 2. been cast on the wider applicability of these methods by the finding that it is impossible to distinguish unambiguously between two semitransparent objects (neither of which is totally transparent) without a certain non-zero probability that the objects absorb a photon [3].

In this note we explore a different problem from that considered in [3] which is probably more important in practice. We consider the case where one wants to determine the transparency of an object with high resolution while minimizing the number of photons absorbed by the object. We shall show in this case that, except for certain specialised tasks, using the quantum mechanical properties of light does not offer significant advantages over more traditional schemes such as counting absorbed photons or simple interferometric procedures that use one photon at a time.

The general scenario that we have in mind is an object which is characterized by a position-dependent transparency $\alpha(x)$. Here $x$ is a coordinate on the surface of the object, and $\alpha$ is the complex amplitude for a photon not to be absorbed by the object. The aim is determine $\alpha(x)$ to high resolution while minimizing the number of photons absorbed by the object. A "minimal absorption measurement" is a protocol whose outcome (a set of measurement results) achieves this aim.

This scenario is difficult to analyze in full generality, and hence we simplify it. As a first step, we consider an extreme simplification in which (a) the object consists of only one pixel (i.e. $x$ takes only one value), and (b) there are only two possible objects. That is, the transparency of the single pixel can take only two values, $\alpha_{1}$ or $\alpha_{2}$. The task is then to determine which object is present (ie. to determine whether the transparency is $\alpha_{1}$ or $\alpha_{2}$ ) while absorbing as few photons as possible. We give a bound on the minimum number of photons that must be absorbed if objects are to be distinguished with high probability. This bound is valid for an arbitrary quantum protocol. In the conclusion we mention how this result can be extended to the case where the transparencies can take a continuous range of values. 
To state our result in the single pixel case, let $\alpha=\left(\alpha_{1}+\alpha_{2}\right) / 2$ be the average of $\alpha_{1}$ and $\alpha_{2}$, and let $\beta_{1}, \beta_{2}$ be the amplitudes that objects 1 and 2 absorb a photon. Let $\beta=\sqrt{1-|\alpha|^{2}}$, so $\beta$ is approximately the average of $\left|\beta_{1}\right|$, $\left|\beta_{2}\right|$. We are interested in the case where the difference of transparencies is small, so we can write $\alpha_{1}=\alpha-\epsilon$ and $\alpha_{2}=\alpha+\epsilon$, where $\epsilon$ is a small (complex) number satisfying $|\epsilon| \ll \frac{\beta^{2}}{2|\alpha|}$. Let $P_{E}$ denote the probability of making a mistake in identifying the object. Denote by $\bar{N}_{i}^{a b s}$ the mean number of absorbed photons in a minimal absorption measurement if object $i$ is to be correctly identified with probability greater than $1-P_{E}$. Then our main result is the following constraint on $\bar{N}_{1}^{a b s}$ and $\bar{N}_{2}^{a b s}$ :

$$
\frac{\bar{N}_{1}^{a b s}+\bar{N}_{2}^{a b s}}{2} \geq \frac{\beta^{4}\left(1-2 \sqrt{P_{E}\left(1-P_{E}\right)}\right)}{2|\epsilon|^{2}}+O(1) .
$$

The most important aspect of (11) is that the mean number of absorbed photons increases as $1 /|\epsilon|^{2}$. In most cases this bound is comparable to the resolution that can be obtained with very simple schemes which do not exploit the full range of possibilities offered by quantum mechanics. Indeed simply counting the number of photons absorbed by the object gives a resolution comparable to the bound of (1), except in the special case where $\alpha_{1}$ and $\alpha_{2}$ differ only in phase.

When $\alpha_{1}$ and $\alpha_{2}$ differ only in phase one must use an interferometric protocol. A simple interferometric protocol, in which the photons can either pass through the object or not do so, yields a $1 / \epsilon^{2}$ dependence of the mean number of absorbed photons, as in (11). However we have only been able to attain the same fourth power dependence on $\beta$ as in (11) by modifying the interferometric protocol in such a way that the photon can either pass a large number $k$ of times through the object or not pass through it at all.

As a further step, we consider the situation where one aims to distinguish not just the transparencies of one pixel but different patterns of transparency $\alpha(x)$ on a set of pixels. We obtain in this case a bound on the minimum number of photons that must be absorbed if objects are to be distinguished with high probability which is similar to the bound obtained in the single pixel case.

We illustrate the multi-pixel situation by an example of a special discrimination task, where a significant decrease in the number of absorbed photons can be obtained by using interferometric protocols that address all the pixels simultaneously, as compared to addressing each pixel individually. This example is based on recent work of Wim van Dam [6]. All transparencies are assumed to be real, i.e. the pixels differ only by the probability that they absorb a photon. We show that a collective measurement of all the pixels decreases the number of absorbed photons, relative to measurements on pixels one by one, by a factor of $\log$ (Number of pixels). Similar decreases in the number of absorbed photons when imaging a multi-pixel object have been obtained independently by Adrian Kent and David Wallace [8].

Finally, we consider some examples which illustrate the limitations of our results. One example is provided by absorption-free measurements in the case $\alpha_{1}=1$ where small differences can be resolved with negligible photon absorption. A second is based on Grover's algorithm [7], which allows essentially absorption-free discrimination in some special situations. We explain why these examples escape the limitations of our bound (1).

The paper is organized as follows. The next three sections are concerned with the case of a single pixel and only two objects. Specifically sections III and III present protocols that yield photon-absorption numbers of the same order as our bound (1), and section IV contains the proof of this bound. Sections $\mathrm{V}$ and VI are concerned with the general case of a multi-pixel image and many possible objects. Section $\mathrm{V}$ obtains an analogous bound to inequality (11) in this more general case, and section VI describes our example in which collective addressing yields a decrease in the number of absorbed photons. Section VII discusses the limitations of our approach, with some examples, and section VIII contains our conclusions and discusses possible extensions of our results.

\section{COUNTING THE NUMBER OF ABSORBED PHOTONS}

In this section and the next two, we consider the case where we have a single pixel, and the transparency of this pixel can take only one of two values, $\alpha_{1}$ or $\alpha_{2}$. Let us suppose that one sends, one by one, $N$ photons through the unknown object and counts how many pass through it without being absorbed. For object $i(i=1,2)$, this number is distributed according to a binomial of mean $\mu_{i}=\left|\alpha_{i}\right|^{2} N$ and standard deviation $\sigma \simeq|\alpha \beta| \sqrt{N}$. The decision strategy that minimizes the probability of error is to compute for which object the observed number of transmitted photons (denoted by $N^{T}$ ) is most likely, and to guess that that is the correct object. Thus there will be some value $N^{T *}$ (with $\left.\mu_{1} \leq N^{T *} \leq \mu_{2}\right)$ such that if $N^{T} \leq N^{T *}$ one guesses that $i=1$ and if $N^{T}>N^{T *}$ one guesses that $i=2$ (we have taken $\left.\left|\alpha_{1}\right|<\left|\alpha_{2}\right|\right)$.

For small error probability $P_{E}$, the number of photons sent through the object $N$ must be large and the two binomials will tend to Gaussians. $N^{T *}$ is then approximately equal to $\frac{\mu_{1}+\mu_{2}}{2}$ and the probability of error is approximately 
$P_{E} \simeq \frac{1}{2}\left(1-\operatorname{erf}\left(\frac{\mu_{2}-\mu_{1}}{\sqrt{8} \sigma}\right)\right)$. This gives $\left(\mu_{2}-\mu_{1}\right) / 2 \sigma=\sqrt{2} \gamma\left(P_{E}\right)$, where $\gamma\left(P_{E}\right)=\operatorname{erf}^{-1}\left(1-2 P_{E}\right)$ (so, for instance, $\gamma=0.91$ when $P_{E}=0.1$, or $\gamma=1.65$ when $P_{E}=0.01$ ). Hence the mean number of absorbed photons for this protocol is

$$
\bar{N}^{a b s}=\beta^{2} N \simeq \frac{2 \beta^{4}|\alpha|^{2} \gamma\left(P_{E}\right)^{2}}{(\alpha \bar{\epsilon}+\bar{\alpha} \epsilon)^{2}}
$$

In the above procedure we have supposed that the photons are sent one by one and that we have a perfect single photon detector at our disposal. We could also have sent a state containing $N$ identical photons and measured the number of transmitted photons. This supposes that one can prepare photon number states and measure photon number. In practice it is of course much easier to send as input a coherent state of amplitude $A$ and measure the intensity transmitted through the object. In this case one finds that $\bar{N}^{a b s} \geq \beta^{2}|\alpha|^{2} \gamma^{\prime}\left(P_{E}\right)^{2} /(\alpha \bar{\epsilon}+\bar{\alpha} \epsilon)^{2}$ where $\gamma^{\prime}\left(P_{E}\right)$ is the analogue of $\gamma$ for a Poisson distribution. It is interesting to note that these two procedures differ in the value of the pre-factor $\left(\beta^{4}\right.$ or $\left.\beta^{2}\right)$, the lesser efficiency of using coherent states presumably being due to the uncertain photon number for such states. We note that since all the other protocols discussed in this article use linear optical elements (beam splitters, phase shifters) they can also be implemented using coherent light. In these cases one also expects an increase in the mean number of absorbed photons for the reason just mentioned.

Returning to inequality (2), when $\alpha$ and $\epsilon$ are real this reduces to

$$
\bar{N}^{a b s} \simeq \frac{\beta^{4} \gamma\left(P_{E}\right)^{2}}{2 \epsilon^{2}},
$$

so in this real case $\bar{N}^{a b s}$ is proportional to $1 /|\epsilon|^{2}$, as in (11). For complex $\alpha$ and $\epsilon$, the denominator in (2) may be much smaller than $|\epsilon|^{2}$; in particular, if $\left|\alpha_{1}\right|=\left|\alpha_{2}\right|$, so $\alpha_{1}$ and $\alpha_{2}$ differ only in phase, or equivalently if $\alpha \bar{\epsilon}+\bar{\alpha} \epsilon=0$, then $\bar{N}^{a b s}=\infty$, and photon-counting fails. In this situation we need an interferometric procedure that is sensitive to the phase of $\alpha$ and $\epsilon$.

\section{INTERFEROMETRIC PROCEDURES}

We focus on the case where $\left|\alpha_{1}\right|=\left|\alpha_{2}\right|$, so simple counting of absorbed photon fails. In this case, one can write $\alpha_{1,2}=\alpha e^{ \pm i \eta}$, where $\alpha$ is real (any global phase can be removed by an appropriate phase shifter placed on the path of the photon sent through the object). Note that $|\beta|^{2}=1-\alpha^{2}=1-\left|\alpha_{1,2}\right|^{2}$ since $\alpha_{1,2}$ differ only by a phase. For $\eta$ small, this can be rewritten as $\alpha_{1,2} \simeq \alpha \pm i \alpha \eta=\alpha \pm i \epsilon$, where $\epsilon=\alpha \eta$.

A simple interferometric procedure to detect object 1 or 2 consists of a Mach-Zender interferometer with the object located in one of the arms. The transmission and reflection amplitudes at the first beam-splitter are $1 / \sqrt{2}$, while at the second beam-splitter the transmission amplitude is $1 / \sqrt{2}$ and the reflection amplitude $i / \sqrt{2}$. A photon originally in the state $|0\rangle$ is sent to $(|0\rangle+|1\rangle) / \sqrt{2}$ by the first beam-splitter. This state then becomes $\left(|0\rangle+\alpha_{i}|1\rangle\right) / \sqrt{1+\left|\alpha_{i}\right|^{2}}$ through interaction with the object. Finally, after the second beam-splitter, the probability of detecting the photon in arm 0 with object $i$ is $\chi_{i}=\left|1+i \alpha_{i}\right|^{2} / 2\left(1+\left|\alpha_{i}\right|^{2}\right)$.

The idea is to send $N$ photons one by one through the interferometer, count the number of times the detection of a photon in arm 0 occurs, and to distinguish objects 1 and 2 by these counts. The mean number of measurement outcome is $\mu_{i}=N \chi_{i}$, so that $\mu_{2}-\mu_{1} \simeq 2 N \epsilon /\left(1+\alpha^{2}\right)$. The standard deviation of the distribution of these counts is $\sigma \simeq \sqrt{N \chi(1-\chi)}$, where $\chi=|1+i \alpha|^{2} / 2\left(1+\alpha^{2}\right)=1 / 2$. Applying the same criterion for distinguishability as in section II one finds $\bar{N}^{a b s}=\gamma^{2} \beta^{2}\left(1+\alpha^{2}\right) / 2 \epsilon^{2}$, so $\bar{N}^{a b s}$ is again proportional to $1 /|\epsilon|^{2}$.

Note, however, that there is a factor of $\beta^{2}$ missing, compared to the bound, $\bar{N}^{a b s} \geq O\left(\beta^{4} /|\epsilon|^{2}\right)$ of (1), and this means that the above simple interferometric method diverges from the multi-photon bound as $\alpha$ tends to 1 and $\beta$ to zero. To recover the correct order in $\beta$, we simply let the photon pass $k$ times through the object before the second beam-splitter instead of just once. The probability of detecting it in arm 0 is then $\chi_{i}=\left|1+i\left(\alpha_{i}\right)^{k}\right|^{2} / 2\left(1+\left|\alpha_{i}\right|^{2 k}\right)$. Counting the number of times detection occurs gives $\mu_{2}-\mu_{1} \simeq 2 N k \alpha^{k-1} \epsilon /\left(1+\alpha^{2 k}\right)$ and $\chi \simeq 1 / 2$. We are interested in the case where $\alpha$ is close to 1 . Writing $\alpha=1-\delta$, with $\delta$ small, and taking $k=1 / \delta$, we find $\mu_{2}-\mu_{1} \simeq 2 N \epsilon / \delta\left(e+e^{-1}\right)$. The total absorption probability is $\frac{1}{2}\left(1-\alpha^{2 k}\right) \rightarrow \frac{1}{2}\left(1-e^{-2}\right)$. Thus $\bar{N}^{a b s} \simeq O\left(\delta^{2} / \epsilon^{2}\right)$, and since $\beta^{2}=1-(1-\delta)^{2} \simeq 2 \delta$ this gives $\bar{N}^{a b s} \simeq O\left(\beta^{4} /|\epsilon|^{2}\right)$, which is the same order as the bound, (1) , in both $\epsilon$ and $\beta$.

Thus we can use simple counting of absorbed photons to achieve the quantum bound, except when $\alpha_{1}$ and $\alpha_{2}$ differ only in phase, in which case an interferometric procedure yields a mean number of absorbed photons of the same order as the bound (11). We now turn to the proof of inequality (11). 


\section{BOUNDS FOR MINIMAL ABSORPTION MEASUREMENTS}

Absorption-free measurement schemes use a single photon and a choice of paths for that photon: through the arm of the interferometer where the object is located, or through the other 'ancillary' arm. However, these protocols are not the most general ones. Indeed, if a single photon is absorbed the protocols stop. But since we are not considering absorption-free measurements but rather minimal absorption schemes we should allow for the possibility that some of the photons may be absorbed while the remaining photons are still available for further operations. We can then imagine protocols where we send a superposition of states with different photon numbers through the object, and possibly perform collective measurements on these photons. Or we can use ancillas which do not interact with the object to create entangled state such as $\mid 2$ photons not passing through the object $\rangle \mid 0$ photons passing through the object $\rangle+$ $\mid 0$ photons not passing through the object $\rangle \mid 2$ photons passing through the object $\rangle$, and all sorts of combinations of this kind.

In order to give a bound on the efficiency of these quantum protocols, we must give a completely general formulation of such protocols. To this end, we divide the total Hilbert space into the product of three subspaces $H_{A} \otimes H_{P} \otimes H_{O}$. The first subspace, $H_{A}$, defines the state of the ancilla. It can be arbitrary; for instance it can be a subspace of the Fock space of the electromagnetic field. The second subspace, $H_{P}$, describes the photons which are sent through the object. A basis of $H_{P}$ is therefore the photon number states $|n\rangle_{P}$. The last subspace, $H_{O}$, corresponds to the state of the object. If $n_{1}, \ldots n_{j}, \ldots$ photons have been absorbed by the object at stages $1, \ldots, j, \ldots$ of the protocol, the state of the object becomes $\left|n_{1}, \ldots, n_{j}, \ldots\right\rangle_{O}$ and represents the state induced in the object by the absorptions. That part of the object Hilbert space that is of interest to us can therefore be written as the tensor product of Fock spaces: $H_{O}^{1} \otimes H_{O}^{2} \otimes \cdots$, where number states in $H_{O}^{j}$ count the number of photons that have been absorbed at stage $j$. The object can also have internal degrees of freedom, which we do not write explicitly.

We now adopt the general formulation of a protocol used in [3]. It was assumed there that a protocol starts from a specified initial state, after which there is a succession of steps, called 'interaction steps', where the photons are sent through the object, alternating with steps where some (arbitrary) unitary transformation occurs. Finally some measurement is made which seeks to determine which object was present. In our general minimal absorption protocol, we assume there is an initial state of the ancilla and photons, $\left|\Psi_{0}\right\rangle_{A P}$, with the object in state $\left|0_{1}, 0_{2}, \ldots\right\rangle_{O}$ since no interaction has yet taken place. The unitary transformation following the $j$-th interaction step is of the form $U_{A P}^{j} \otimes I_{O}$.

In [3] the interaction step is assumed to consist of two parts: a unitary transformation which describes the initial interaction between photon and object, and a measurement which describes the subsequent collapse of the photon/object system into 'interacted' or 'non-interacted' outcomes (the object is assumed to be macroscopic). Here we assume that each interaction step consists only of a unitary transformation, and we do not carry out the collapse step. If we were to trace over the object space $H_{O}$ at the end of the protocol, before the final measurement, this would be equivalent to the complete interaction step in [3]. Instead, however, we assume for mathematical simplicity that the final measurement is completely arbitrary and can also act on the state of the object. This can only increase the information gain and hence the efficiency of the protocol. For this reason the bound given by inequality (11) is probably not optimal.

We assume the interaction step takes the form of a unitary transformation $I_{A} \otimes U_{P O}^{i n t}$, where $I_{A}$ is the identity on the ancilla Hilbert space. The action of $U_{P O}^{i n t}$ is given by

$$
a_{P}^{\dagger} \rightarrow \alpha a_{P}^{\dagger}+\beta b_{j_{O}}^{\dagger}
$$

where $a_{P}^{\dagger}$ and $b_{j O}^{\dagger}$ are the creation operators in $H_{P}$ and $H_{O}^{j}$, respectively. The evolution that this induces on the state $|a\rangle_{A}|1\rangle_{P}\left|0_{j}\right\rangle_{O}=a_{P}^{\dagger}|a\rangle_{A}|0\rangle_{P}\left|0_{j}\right\rangle_{O}$, which represents a single photon sent through the object (and an ancilla), is

$$
|a\rangle_{A}|1\rangle_{P}\left|0_{j}\right\rangle_{O} \rightarrow \alpha|a\rangle_{A}|1\rangle_{P}\left|0_{j}\right\rangle_{O}+\beta|a\rangle_{A}|0\rangle_{P}\left|1_{j}\right\rangle_{O}
$$

If $l$ photons are sent through the object, the unitary evolution (4) gives

$$
\begin{aligned}
|a\rangle_{A}|l\rangle_{P}\left|0{ }_{j}\right\rangle_{O}=\frac{\left(a_{P}^{\dagger}\right)^{l}}{\sqrt{l !}}|a\rangle_{A}|0\rangle_{P}\left|0_{j}\right\rangle_{O} \rightarrow & \frac{\left(\alpha a_{P}^{\dagger}+\beta b_{j_{O}}^{\dagger}\right)^{l}}{\sqrt{l !}}|a\rangle_{A}|0\rangle_{P}\left|0{ }_{j}\right\rangle_{O} \\
& =\sum_{m=0}^{l}\left(\begin{array}{c}
l \\
m
\end{array}\right)^{1 / 2} \alpha^{m} \beta^{l-m}|a\rangle_{A}|m\rangle_{P}\left|(l-m)_{j}\right\rangle_{O} \\
& =|a\rangle_{A}\left|\widetilde{l}_{j}\right\rangle_{P O} .
\end{aligned}
$$

In the last line we have introduced the notation

$$
\left|\widetilde{l}_{j}\right\rangle_{P O}=\sum_{m=0}^{l}\left(\begin{array}{c}
l \\
m
\end{array}\right)^{1 / 2} \alpha^{m} \beta^{l-m}|m\rangle_{P}\left|(l-m)_{j}\right\rangle_{O}
$$


that will serve us later.

We use the following notation for the state function, given transparency $i$, during successive stages of the protocol:

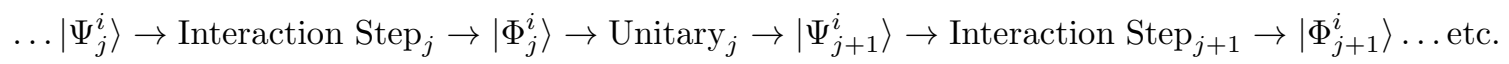

With this notation, we can explicitly write the state immediately before the $j$-th interaction step as

$$
\left|\Psi_{j}^{i}\right\rangle_{A P O}=\sum_{k, l, n_{1}, \ldots n_{j-1}} C_{k l n_{1}, \ldots n_{j-1}}^{j, i}|k\rangle_{A}|l\rangle_{P}\left|n_{1}, \ldots n_{j-1}, 0_{j}, 0_{j+1}, \ldots\right\rangle_{O}
$$

where $\left\{|k\rangle_{A}, k=0 \ldots S\right\}$ is a basis in $H_{A}$ and $\left\{|l\rangle_{P}\right\}$ are the Fock states in $H_{P}$.

Immediately after the interaction step, $\left|\Psi_{j}^{i}\right\rangle_{A P O}$ becomes

$$
\begin{aligned}
\left|\Phi_{j}^{i}\right\rangle_{A P O} & =I_{A} \otimes U_{P O}^{i n t}\left|\Psi_{j}^{i}\right\rangle_{A P O} \\
& \left.=\sum_{k, l, n_{1}, \ldots n_{j-1}} C_{k l n_{1}, \ldots n_{j-1}}^{j, i}|k\rangle_{A} \widetilde{l}_{j}^{i}\right\rangle_{P O}\left|n_{1}, \ldots n_{j-1}, 0_{j+1}, \ldots\right\rangle
\end{aligned}
$$

where we have used the notation of (6).

After this interaction step, the unitary transformation $U_{A P} \otimes I_{O}$ transforms $\left|\Phi_{j}^{i}\right\rangle_{A P O}$ to $\left|\Psi_{j+1}^{i}\right\rangle_{A P O}$. Following [3], we define

$$
f_{j}=\left|\left\langle\Psi_{j}^{1} \mid \Psi_{j}^{2}\right\rangle\right|
$$

The overlap $f_{j}$ plays an important part in our argument. It measures how much the two states corresponding to evolution with the two transparencies $i=1,2$ differ, and thus how easily one can distinguish them. The smaller this quantity, the more efficient the protocol.

Unitarity implies that

$$
f_{j+1}=\left|\left\langle\Phi_{j}^{1} \mid \Phi_{j}^{2}\right\rangle\right|
$$

which, using the normalization conditions

$$
\begin{aligned}
& { }_{A}\left\langle k \mid k^{\prime}\right\rangle_{A}=\delta_{k k^{\prime}}, \\
& { }_{O}\left\langle n_{1} \ldots n_{j-1} \mid n_{1}^{\prime} \ldots n_{j-1}^{\prime}\right\rangle_{O}=\delta_{n_{1} n_{1}^{\prime}} \ldots \delta_{n_{j-1} n_{j-1}^{\prime}},
\end{aligned}
$$

can be written as

$$
f_{j+1}=\left|\sum_{k, l, l^{\prime}, n_{1}, \ldots n_{j-1}}\left(\bar{C}_{l}^{j, 1} C_{l^{\prime}}^{j, 2}\right)_{k n_{1} \ldots n_{j-1} P O}\left\langle\widetilde{l}_{j}^{1} \mid \widetilde{l}_{j}^{2}\right\rangle_{P O}\right| .
$$

Using (6), we can compute ${ }_{P O}\left\langle\tilde{l}_{j}^{1} \mid \tilde{l}_{j}^{\prime 2}\right\rangle_{P O}$ :

$$
{ }_{P O}\left\langle\tilde{l}_{j}^{1} \tilde{l}_{j}^{\prime 2}\right\rangle_{P O}=\delta_{l l^{\prime}} \sum_{m=0}^{l}\left(\begin{array}{c}
l \\
m
\end{array}\right)\left(\bar{\alpha}_{1} \alpha_{2}\right)^{m}\left(\bar{\beta}_{1} \beta_{2}\right)^{l-m}=\delta_{l l^{\prime}}\left(\bar{\alpha}_{1} \alpha_{2}+\bar{\beta}_{1} \beta_{2}\right)^{l} .
$$

We can then rewrite (7) as

$$
f_{j+1}=\left|\sum_{k, l, n_{1}, \ldots n_{j}}\left(\bar{C}^{j, 1} C^{j, 2}\right)_{k l n_{1} \ldots n_{j}}\left(\bar{\alpha}_{1} \alpha_{2}+\bar{\beta}_{1} \beta_{2}\right)^{l}\right| .
$$

We now obtain an approximation for the term $\bar{\alpha}_{1} \alpha_{2}+\bar{\beta}_{1} \beta_{2}$. The assumption we are making that the final measurement can act on the state of the object is physically incorrect. We should be tracing over the Hilbert space of the object before the final measurement, in which case the phases of the absorption amplitudes $\beta_{i}$ would be irrelevant. We are therefore free to choose the phases of the $\beta_{i}$ as we wish, so as to obtain the best bound. Suppose therefore that

$$
\bar{\beta}_{1} \beta_{2}=e^{i \phi} \sqrt{1-\bar{\alpha}_{1} \alpha_{1}} \sqrt{1-\bar{\alpha}_{2} \alpha_{2}},
$$


where $\phi$ is real but otherwise can be chosen freely. By adjusting $\phi$ appropriately, with the assumption $|\epsilon| \ll \beta^{2}$, we can make $\bar{\alpha}_{1} \alpha_{2}+\bar{\beta}_{1} \beta_{2}$ real. Indeed, we can rewrite $\bar{\alpha}_{1} \alpha_{2}+\bar{\beta}_{1} \beta_{2}$ as $e^{i\left(\rho_{1}-\rho_{2}\right)} \cos \theta_{1} \cos \theta_{2}+e^{i \phi} \sin \theta_{1} \sin \theta_{2}$, where $\cos \theta_{i}=\left|\alpha_{i}\right|$ and where $\rho_{i}$ is the phase of $\alpha_{i}$. The imaginary terms vanish if $\sin \phi=\cos \theta_{1} \cos \theta_{2} \sin \left(\rho_{2}-\rho_{1}\right) /\left(\sin \theta_{1} \sin \theta_{2}\right)$. This is possible only if the right hand side is $\leq 1$, which is ensured if $|\epsilon|<\frac{\beta^{2}}{2|\alpha|}$.

With this choice of $\phi$, we find

$$
\bar{\alpha}_{1} \alpha_{2}+\bar{\beta}_{1} \beta_{2}=1-\frac{2|\epsilon|^{2}}{\beta^{2}}+O\left(\epsilon^{4}\right)
$$

Introducing the notation

$$
\left(\bar{\alpha}_{1} \alpha_{2}+\bar{\beta}_{1} \beta_{2}\right)^{l}=1-\delta_{l},
$$

eq (9) implies that

$$
\delta_{l} \leq l\left(\frac{2|\epsilon|^{2}}{\beta^{2}}+O\left(\epsilon^{4}\right)\right)
$$

since $(1-x)^{l} \geq 1-l x$, for $0 \leq x \leq 1$ (as can easily be verified by induction on $l$ ).

Turning back to eq (8) we are now able to compute $f_{j+1}$ :

$$
\begin{aligned}
f_{j+1} & =\left|\sum_{k, l, n_{1}, \ldots n_{j}}\left(\bar{C}^{j, 1} C^{j, 2}\right)_{k l n_{1} \ldots n_{j}}\left(\bar{\alpha}_{1} \alpha_{2}+\bar{\beta}_{1} \beta_{2}\right)^{l}\right| \\
& =\left|\sum_{k, l, n_{1}, \ldots n_{j}}\left(\bar{C}^{j, 1} C^{j, 2}\right)_{k l n_{1} \ldots n_{j}}\left(1-\delta_{l}\right)\right| \\
& \geq\left|\sum_{k, l, n_{1}, \ldots n_{j}}\left(\bar{C}^{j, 1} C^{j, 2}\right)_{k l n_{1} \ldots n_{j}}\right|-\left|\sum_{k, l, n_{1}, \ldots n_{j}}\left(\bar{C}^{j, 1} C^{j, 2}\right)_{k l n_{1} \ldots n_{j}} \delta_{l}\right| \\
& \geq f_{j}-\sum_{k, l, n_{1}, \ldots n_{j}}\left|\left(\bar{C}^{j, 1} C^{j, 2}\right)_{k l n_{1} \ldots n_{j}} \delta_{l}\right| \\
& =f_{j}-\left(\frac{2|\epsilon|^{2}}{\beta^{2}}+O\left(\epsilon^{4}\right)\right) \sum_{k, l, n_{1}, \ldots n_{j}}\left|\left(\bar{C}^{j, 1} C^{j, 2}\right)_{k l n_{1} \ldots n_{j}}\right| l \\
& \geq f_{j}-\left(\frac{2|\epsilon|^{2}}{\beta^{2}}+O\left(\epsilon^{4}\right)\right) \sum_{k, l, n_{1}, \ldots n_{j}} \frac{\left|C_{k l n_{1} \ldots n_{j}}^{j, 1}\right|^{2}+\left|C_{k l n_{1} \ldots n_{j}}^{j}\right|^{2}}{2} l \\
& =f_{j}-\left(\frac{2|\epsilon|^{2}}{\beta^{2}}+O\left(\epsilon^{4}\right)\right)\left(n_{j}^{1}+n_{j}^{2}\right) / 2
\end{aligned}
$$

In the last equality, $n_{j}^{i}=\sum_{k, l, n_{1}, \ldots n_{j}}\left|C^{j, i}\right|_{k l n_{1} \ldots n_{j}}^{2} l$ is the average number of photons sent through the object at interaction step $j$. Starting from $f_{1}=1$, and iterating the formula above, we conclude that

$$
f_{K} \geq 1-\left(\frac{2|\epsilon|^{2}}{\beta^{2}}+O\left(\epsilon^{4}\right)\right) \sum_{j=1}^{K-1} \frac{n_{j}^{1}+n_{j}^{2}}{2}=1-\left(\frac{2|\epsilon|^{2}}{\beta^{2}}+O\left(\epsilon^{4}\right)\right) \frac{N^{1}+N^{2}}{2},
$$

$N^{i}$ being the total number of photons that are sent through object $i$ in the protocol. The average number of photons absorbed by object $i$ is $\bar{N}_{i}^{a b s}=\left|\beta_{i}\right|^{2} N^{i}$, so that

$$
\frac{\bar{N}_{1}^{a b s}+\bar{N}_{2}^{a b s}}{2}=\frac{\left|\beta_{1}\right|^{2} N^{1}+\left|\beta_{2}\right|^{2} N^{2}}{2} \simeq \beta^{2} \frac{N^{1}+N^{2}}{2}
$$

where the last approximation is valid if $|\epsilon| \ll \frac{\beta^{2}}{2|\alpha|}$. From (11), we thus find that

$$
\frac{\bar{N}_{1}^{a b s}+\bar{N}_{2}^{a b s}}{2} \geq \frac{\beta^{4}}{2|\epsilon|^{2}}\left(1-f_{K}\right)+O\left(\epsilon^{0}\right) .
$$

By choosing measurement axes symmetrical with respect to the final state vectors $\Psi_{K}^{1}$ and $\Psi_{K}^{2}$, one obtains the minimal probability of mis-identification of the object, $P_{E}=\frac{1}{2}\left(1-\sqrt{1-f_{K}^{2}}\right)$, which implies $f_{K}=2 \sqrt{P_{E}\left(1-P_{E}\right)}$ [9]. Putting everything together gives our bound (11).

\section{DISCRIMINATING FAINT IMAGES}

We now consider the more general situation where we have $M$ pixels, and $L$ images, i.e. assignments of transparencies to these pixels. The transparencies at any one pixel for all the different images are assumed to be close. We 
obtain a bound on the mean number of absorbed photons that is similar to inequality (11), with $\epsilon$ replaced by the maximum difference between the transparencies of individual pixels. Because this bound does not decrease with the number of pixels, it is not obvious that collective addressing of the pixels offers a significant advantage over addressing each pixel individually. Nevertheless we shall give in the next section an example where such collective addressing significantly decreases the mean number of absorbed photons.

The state at stage $j$ when there are $M$ pixels and $L$ images can be written as

$$
\left|\psi_{j}^{p}\right\rangle_{A P O}=\sum C_{k, l_{1}, \ldots, l_{M} ; n_{11}, n_{12}, \ldots, n_{1 M}, n_{21}, \ldots, n_{j-1 M}}^{j, p}|k\rangle_{A}\left|l_{1}\right\rangle \ldots\left|l_{M}\right\rangle\left|n_{11}, \ldots\right\rangle,
$$

where $p, 1 \leq p \leq L$ specifies the image, $\left\{|k\rangle_{A}, k=0 \ldots S\right\}$ is a base in $H_{A}, l_{i}$ is the number of photons sent through pixel $i$ and $n_{a b}$ is the number of photons absorbed at stage $a$ by pixel $b$. We can write the coefficient concisely as $C_{k, \mathbf{1}, \mathbf{n}}^{j, p}$.

If $f_{j}^{p, q}$ denotes the absolute value of the overlap between states with image $p$ and $q$ at stage $j$, the analogue of eq. (8) is

$$
f_{j+1}^{p, q}=\left|\left\langle\psi_{j+1}^{p} \mid \psi_{j+1}^{q}\right\rangle\right|=\left|\sum_{k, \mathbf{l}, \mathbf{n}}\left(\bar{C}^{j, p} C^{j, q}\right)_{k, \mathbf{l}, \mathbf{n}} \prod_{i=1}^{M}\left(\bar{\alpha}_{i}^{p} \alpha_{i}^{q}+\bar{\beta}_{i}^{p} \beta_{i}^{q}\right)^{l_{i}}\right|,
$$

where $\alpha_{i}^{p}$ is the amplitude for absorbing a photon at pixel $i$ given image $p$. Assuming that $\epsilon_{i}^{p, q}=\left(\alpha_{i}^{p}-\alpha_{i}^{q}\right) / 2$ is small, for any $i$ and $p, q$, we can write this approximately as

$$
f_{j+1}^{p, q} \simeq\left|\sum\left(\bar{C}^{j, p} C^{j, q}\right)_{k, \mathbf{l}, \mathbf{n}}\left(1-\sum_{i} \delta_{l_{i}}^{i}\right)\right|
$$

where $\delta_{l}^{i} \leq l\left(2\left|\epsilon_{i}^{p, q}\right|^{2} / \beta_{i}^{2}+O\left(\left(\epsilon_{i}^{p, q}\right)^{4}\right)\right)$, and $\beta_{i}$ is the average of $\left|\beta_{i}^{p}\right|$ over all images $p$. Leaving out the $O\left(\epsilon^{4}\right)$ terms, the steps leading to eq. (10) and (11) then yield

$$
\sum_{k, \mathbf{l}, \mathbf{n}} \sum_{i=1}^{M} \frac{\left|C_{k, \mathbf{l}, \mathbf{n}}^{j, p}\right|^{2}+\left|C_{k, \mathbf{l}, \mathbf{n}}^{j, q}\right|^{2}}{2} l_{i} \frac{2\left|\epsilon_{i}^{p, q}\right|^{2}}{\beta_{i}^{2}} \geq 1-f_{K}^{p, q} .
$$

or

$$
\sum_{i=1}^{M}\left(N_{i}^{p}+N_{i}^{q}\right) \frac{\left|\epsilon_{i}^{p, q}\right|^{2}}{\beta_{i}^{2}} \geq 1-f_{K}^{p, q}
$$

where $N_{i}^{p}$ is the number of photons passing through pixel $i$ under assignment $p$. Now consider the special situation (which will arise in the next section) where the $\alpha_{i}^{p}$ are close in value for all $i$ and $p$, i.e. over all pixels and all images. Let $\beta$ be the average of all the $\left|\beta_{i}^{p}\right| \mathrm{s}$, and let $\left|\epsilon^{p, q}\right|=\max _{i}\left\{\left|\epsilon_{i}^{p, q}\right|\right\}$ a. Then we have the corollary to (13)

$$
\bar{N}_{p}^{a b s}+\bar{N}_{q}^{a b s} \geq \frac{\beta^{4}\left(1-f_{K}^{p, q}\right)}{\left|\epsilon^{p, q}\right|^{2}}
$$

where $\bar{N}_{p}^{a b s}$ is the mean number of photons absorbed if the image is $p$. In the case where we wish to discriminate between two images, $p$ and $q$, we obtain the analogue of inequality (11):

$$
\frac{\bar{N}_{p}^{a b s}+\bar{N}_{q}^{a b s}}{2} \geq \frac{\beta^{4}\left(1-2 \sqrt{P_{E}\left(1-P_{E}\right)}\right)}{2\left|\epsilon^{p, q}\right|^{2}},
$$

where $P_{E}$ is the probability of mistaking image $p$ for the image $q$. If the task is to distinguish one image from all $L-1$ others, then averaging over all $p \neq q$ gives

$$
\hat{N}^{a b s} \geq \frac{\beta^{4}\left(1-2 \sqrt{P_{E}\left(1-P_{E}\right)}\right)}{2|\epsilon|^{2}},
$$

where $|\epsilon|=\max _{p, q}\left\{\left|\epsilon^{p, q}\right|\right\}, P_{E}$ now denotes the maximum probability of confusing any two images, and $\hat{N}^{a b s}$ is the mean absorption $N_{p}^{a b s}$ averaged over all images $p$. 


\section{LOGARITHMIC GAIN IN THE NUMBER OF ABSORBED PHOTONS}

The bound (15) is simply the single-pixel bound (1) applied to the pixel $i$ for which the difference of transparencies $\left|\epsilon_{i}^{p, q}\right|=\left|\alpha_{i}^{p}-\alpha_{i}^{q}\right| / 2$ is largest. This bound does not depend on the number of pixels in the image. Nevertheless we shall show that it is possible to decrease the number of absorbed photons by addressing all the pixels collectively, as compared to addressing them one by one.

Wim van Dam [6] considered the following problem: Given a Hadamard matrix $H$, an oracle evaluates the function $f(i)=H_{p i}$, where $H_{p i}$ is the $i$-th element of the $p$-th row of $H$, for some specified $p$. One wishes to know the smallest number of calls to the oracle needed to determine $p$. We consider an analogous problem here. Suppose $\alpha_{i}^{p}=\alpha+\epsilon H_{p i}$, for small $\epsilon$. The task is to determine $p$ with a minimum number of photons absorbed. We can think of the $\epsilon H_{p i}$ term as defining a faint image against the background of $\alpha$. This is an 'epsilon' version of van Dam's problem, with a passage of a photon through the pixels playing the role of a call to an oracle. We assume that $\alpha$ and $\epsilon$ are real.

Suppose the Hadamard matrix is un-normalized, so the entries are \pm 1 , and that the top row of $H$ is all +1 s. Given two rows, $p$ and $q, p \neq q, \epsilon_{i}^{p, q}=0$ at the pixels $i$ where the rows $p$ and $q$ agree, and $\epsilon_{i}^{p, q}=\epsilon$ otherwise. Thus inequality (16) implies

$$
\hat{N}^{a b s} \geq \frac{\beta^{4}(1-f)}{2 \epsilon^{2}}
$$

and $\hat{N}^{a b s}$ is $O\left(1 / \epsilon^{2}\right)$.

Following [6], one can achieve this bound with the following simple quantum algorithm. Assume $p \neq 1$. Prepare the one photon state $|\psi\rangle=\frac{1}{\sqrt{M}} \sum|i\rangle$. After the photon passes through the pixels once, there is a probability $\alpha^{2}+\epsilon^{2}$ of the photon not being absorbed, in which case $|\psi\rangle=\sum\left(\alpha+\epsilon H_{p i}\right)|i\rangle / \sqrt{M\left(\alpha^{2}+\epsilon^{2}\right)}$. Applying the unitary operator $H / \sqrt{M}$ to this gives $(\alpha|1\rangle+\epsilon|p\rangle) / \sqrt{\alpha^{2}+\epsilon^{2}}$, and $|p\rangle$ can be detected with $O\left(1 / \epsilon^{2}\right)$ independent repeats of the process. Thus we get the same order as our bound.

Let us now suppose that we address each pixel individually. Specifically we shall suppose that we send photons one by one through the pixels and measure whether they are absorbed or not. The following informal argument gives a bound on the value for $\bar{N}^{a b s}$ for such an individual-pixel protocol. Let $A=\left\{p_{A}, p_{N}\right\}$ be the distribution with probability $p_{A}$ of absorption and $p_{N}$ of non-absorption at pixel $i$. Let $R=\left\{p_{1}, \ldots, p_{M}\right\}$ be the distribution of probabilities of the Hadamard rows. The mutual information $I(A: R)=H(A)+H(R)-H(A, R)$ tells us how much on average we learn (in bits) on being told whether a photon is absorbed at pixel $i$ or not.

Given prior probabilities $\pi_{p}$ for row $p, H(R)=-\sum_{p} \pi_{p} \log \pi_{p}$. The term $H(A, R)$ is given by

$$
H(A, R)=-\sum_{p}\{P(A, p) \log P(A, p)+P(N, p) \log P(N, p)\},
$$

where $P(X, p)$ is the joint probability of row $p$ being chosen and the photon being absorbed $(X=A)$ or not $(X=N)$. Since $P(A \mid p)=\left(\alpha+H_{p i} \epsilon\right)^{2}$, we have $P(A, p)=P(A \mid p) P(p)=\left(\alpha+H_{p i} \epsilon\right)^{2} \pi_{p}$. Similarly, $P(N, p)=\left\{1-\left(\alpha+H_{p i} \epsilon\right)^{2}\right\} \pi_{p}$. Finally, $H(A)=-p_{A} \log p_{A}-p_{N} \log p_{N}$, where $p_{X}=\sum_{p} P(X, p)$, for $X=A, N$. Up to second order in $\epsilon$ one finds

$$
I(A: R) \simeq \frac{2 \epsilon^{2}}{\beta^{2} \log 2}\left\{\sum_{p} \pi_{p} H_{p i}^{2}-\left(\sum_{p} \pi_{p} H_{p i}\right)^{2}\right\} \leq \frac{2 \epsilon^{2}}{\beta^{2} \log 2} .
$$

As $\log _{2} M$ bits are needed to distinguish one row from $M$ others, and each photon yields at most $4 \epsilon^{2} /\left(\beta^{2} \log 2\right)$ bits, we need at least $N$ photons, where $4 N \epsilon^{2} /\left(\beta^{2} \log 2\right) \geq \log _{2} M$, or $N \geq \beta^{2} \log M /\left(4 \epsilon^{2}\right)$. Thus $N^{a b s} \geq \beta^{4} \log M /\left(4 \epsilon^{2}\right)$. The $\log M$ factor here is the counterpart of the $\log n$ classical bound given in [6], Lemma 5 .

A value of $N^{a b s}$ of this order can be attained by an algorithm in which the same number $N / M$ of photons is sent through each pixel. Denoting by $n_{i}$ the number of photons transmitted at pixel $i$, the algorithm seeks the value of $q$ which maximizes the sum $S_{q}=\sum_{i} H_{q i} n_{i}$ if $q \neq$ top row, $S_{q}=\sum_{i} H_{q i} n_{i}-N \alpha^{2}$ if $q=$ top row. It is easy to check that the distributions for $S_{q}$ have standard deviation $\alpha \beta \sqrt{N}$ and the difference of the means for $p$ and $q \neq p$ is $2 N \alpha \epsilon$. To distinguish one distribution from amongst $M$ we therefore need $\alpha \beta \sqrt{N \log M}=2 N \alpha \epsilon$, implying $\bar{N}_{a b s}=O\left(\log M / \epsilon^{2}\right)$. Thus the collective and individual-pixel bounds can both be attained, and there is a collective gain of a factor of $\log M$. 


\section{BEATING THE BOUNDS: LIMITATIONS OF OUR RESULTS.}

We now give some examples where it is possible to make large gains over simple counting of absorbed photons. These examples may seem to violate our main inequality (1), but we explain why this is not so.

Consider first absorption-free measurement [1]. Given a completely absorbing and a completely transparent object, this offers the possibility of discrimination without any photons being absorbed (with probability approaching one [2]). Of course, our inequality (11) does not apply here, since the transparencies being compared are not close in value. However, in the case where $\alpha_{1}=1$, and $\alpha_{2}$ takes some value not equal to $\alpha_{1}$, essentially absorption-free discrimination is always possible [3]. Suppose we choose $\alpha_{2}=1-2 \epsilon$ for small $\epsilon$. Then $\beta \simeq \sqrt{2 \epsilon}$, and, carrying through the calculations leading to (1), one finds $N^{a b s} \geq\left(1-2 \sqrt{P_{E}\left(1-P_{E}\right)}\right)(1-\epsilon)$. Since the absorption-free measurement gives an arbitrarily small $N^{a b s}$ with $P_{E}=0$, we seem to have a violation of our inequality. However, there is in fact no contradiction, since the condition $|\epsilon| \ll \frac{\beta^{2}}{2|\alpha|}$ is not satisfied, and this is used in the proof of (1).

It is interesting to note that the inequality in [3] for the probability of absorption-free discrimination yields the same order dependence as (11) in the range where they may legitimately be compared. In fact, if one asks how many photons would be absorbed by repeating an absorption-free protocol until it succeeds, one can express this in terms of the probability $P(a b s \mid i)$ of a photon being absorbed during the protocol. One sums over repeated runs with absorptions until no absorption occurs, when the object will be reliably identified. Thus

$$
\bar{N}_{i}^{a b s}=\sum_{k=0}^{\infty} k(1-P(a b s \mid i)) P(a b s \mid i)^{k}=\frac{P(a b s \mid i)}{(1-P(a b s \mid i))}
$$

We know from [3] that $P(a b s \mid 1) P(a b s \mid 2) \geq \eta^{2}$ where $\eta=\frac{\left|\beta_{1} \beta_{2}\right|}{\left|\left(1-\bar{\alpha}_{1} \alpha_{2}\right)\right|}$. With the same assumption as in our proof of (11), i.e. $|\epsilon| \ll \frac{\beta^{2}}{2|\alpha|}$, we find $\frac{\left|\beta_{1} \beta_{2}\right|}{\left|\left(1-\bar{\alpha}_{1} \alpha_{2}\right)\right|} \simeq 1-\frac{2|\epsilon|^{2}}{\beta^{4}}$. There is thus necessarily a value of $i$ so that $P(a b s \mid i) \geq \eta$. This implies that for that value of $i$ we have

$$
\bar{N}_{i}^{a b s} \geq \frac{\eta}{1-\eta} \simeq \frac{\beta^{4}}{2\left|\epsilon^{2}\right|}
$$

with the same dependence on $\beta$ and $\epsilon$ as our bound (11).

We turn now to another example, where collective measurement of many pixels apparently enables our bound to be beaten. This example is inspired by Grover's search algorithm [7]. Recall that the oracle in Grover's algorithm carries out the transformation $|x\rangle|y\rangle \rightarrow|x\rangle|y \oplus f(x)\rangle$ where $x=1, \ldots, M$ is the position, $y=0,1$ and $f(x)=0$ except if $x=x_{0}$ in which case $f\left(x_{0}\right)=1$. This transformation can be mapped into the alternative equivalent form $|x\rangle \rightarrow(-1)^{f(x)}|x\rangle$. It is this second form we shall use below. The aim in Grover's problem is to find $x_{0}$ by addressing the oracle as few times as possible. A classical search algorithm would need the oracle to be addressed $O(M)$ times, whereas Grover's algorithm requires only $O(\sqrt{M})$ calls of the oracle.

Consider an object composed of M pixels. As a first stage suppose that each pixel is completely transparent, but that a single pixel $x_{0}$ induces a phase of -1 , whereas all the other pixels induce a phase of +1 . We are then exactly in the situation of Grover's algorithm, and can determine which pixel induces the anomalous phase in $\sqrt{M}$ passages through the object.

Now suppose that the object has a very small probability $\beta^{2} \ll 1$ of absorbing a photon each time it passes through a pixel. Then the probability that Grover's algorithm succeeds is $|\alpha|^{2 \sqrt{M}} \simeq \exp \left(-\beta^{2} \sqrt{M}\right)$. On the other hand the probability that an algorithm that addresses each pixel individually succeeds without absorbing a single photon is $|\alpha|^{2 M} \simeq \exp \left(-\beta^{2} M\right)$. Thus if $\beta^{-2} \ll M \ll \beta^{-4}$, one can find the anomalous pixel using Grover's algorithm with vanishing probability that a photon is absorbed, whereas addressing each pixel individually would entail a large number of absorbed photons. Note that if the number $M$ of pixels is larger than $\beta^{-4}$, then Grover's algorithm will no longer work because the photon will be absorbed before completion of the algorithm.

We now create an "epsilon" version of this problem by supposing that the anomalous pixel $x_{0}$ induces a phase $e^{i \epsilon}$ relative to all the other pixels. In this case we can either replace the call to the oracle in Grover's algorithm by $n$ successive passages of the photon through the pixels, with $n \epsilon=\pi$; or we can use Fahri and Gutmann's continuous version of Grover's algorithm [11]. In either case the total number of times the photon must pass through the object is $N \simeq \sqrt{M} / \epsilon$. The probability that the photon is absorbed is therefore $P_{a b s} \simeq \beta^{2} \sqrt{M} / \epsilon$. Just as with the limiting case, $\alpha_{1}=1, \alpha_{2}=1-2 \epsilon$, of absorption-free measurement, a violation of (11) seems at first sight possible. However, this is only attainable by having $P_{a b s}$ small, which means that $\beta^{2} \ll \epsilon$, so again the conditions for our inequality are not satisfied. 
Qualitatively, the advantage of taking the object to be extremely transparent is that quantum coherence can be maintained over many passages of a photon through the object. This is illustrated not only by the examples above, but also by the interferometric example at the end of section III, where, in the limit of extreme transparency, it is advantageous to use a protocol in which the photon passes many times through the object. The latter situation gave absorptions consistent with our inequality (1), whereas in the examples considered in this section $\beta$ and $\epsilon$ are of equal (small) magnitude, so the inequality does not apply. It would be interesting to find bounds which hold when both $\beta$ and $\epsilon$ tend to zero.

\section{CONCLUSION}

The possibility of absorption-free measurements [1] suggests that one might be able to use the quantum character of light to significantly improve the imaging of photosensitive objects, where one wants to minimize the number of absorbed photons. However, as we have just seen in the preceding section, discrimination of close values of transparency by repeated trials of absorption-free measurement leads to a bound on the number of absorbed photons compatible with (11).

The conditions under which we have derived (1) are more general than this, since absorption-free measurement uses one photon at a time, whereas we allow protocols with arbitrary numbers of photons. Our result imposes an inescapable bound of order $1 /|\epsilon|^{2}$ on the mean number of absorbed photons. In most cases this bound can be attained by simply sending a certain number of photons through the object and counting the number of transmitted photons. When $\alpha_{1}$ and $\alpha_{2}$ differ only in phase, an interferometric set-up in which each photon can either pass through through the object or take an alternative route also gives rise to a mean number of absorbed photons of order $1 /|\epsilon|^{2}$. However when the probability that the object absorbs a photon tends to zero, it is advantageous to use a modified protocol in which the photon passes through the object many times.

Let us note parenthetically that using classical light as a source is in general disadvantageous, particularly in the limit where the object is very transparent. This seems to be because the photon number is ill-defined for classical light, and this extra noise implies that more photons must be sent through the object compared to a source with well-defined photon number.

Of course, the problem of discriminating two close transparencies is a specialized one. A more realistic problem would be that of putting narrow limits on a transparency that can take a continuous range of values. However, any quantum protocol that achieves the latter goal must select from a number of measurement outcomes, and must therefore be able to discriminate between pairs of outcomes for close transparencies. Our $1 /|\epsilon|^{2}$ bound therefore applies to such protocols, with $\epsilon$ now being the standard deviation of the estimated transparency. In general, counting the number of absorbed photons will require of order $1 /|\epsilon|^{2}$ photons to limit the estimated transparency within a standard deviation of $\epsilon$. Only in the case where the objects have the same probability of absorbing a photon, but differ in the phase they induce, is an interferometric protocol necessary.

Despite these rather negative conclusions for the single pixel case, interferometric protocols can offer a significant advantage when the task is to discriminate patterns of many pixels, and where all the pixels are addressed collectively rather than individually. The example of discrimination between a set of orthogonal patterns (added to a constant background) shows that a $\log M$ gain is possible for $M$ pixels, and Kent and Wallace have shown a gain of the same order in the detection of defective pixels [8]. We have also considered some special cases of absorption-free measurement and searches with Grover's algorithm which do not meet the background assumptions of our inequality (11) and are thereby able to exceed the bounds it imposes. Finding further algorithms that can answer questions about pixel arrays with low absorption cost seems an interesting area for further exploration.

\section{AKNOWLEDGEMENTS}

We thank Adrian Kent for helpful conversations and for drawing our attention to the possibility of a logarithmic gain of quantum multi-pixel algorithms compared to classical ones. We acknowledge financial support by the European Science Foundation. S.M. is a research associate of the Belgian National Research Fund, he acknowledges funding by the European Union under project EQUIP (IST-FET program). 
[1] Elitzur, A. C. and Vaidman, L., Found. of Phys.23, 987-997 (1993).

[2] Kwiat, P. G., Weinfurter, H., Herzog, T., Zeilinger, A. and Kasevich, M. A., Phys.Rev. Lett. 74, 4763-4766 (1995).

[3] G. Mitchison and S. Massar, preprint avaible at http://xxx.lanl.gov/quant-ph/ 0003140, to be published in Phys. Rev. A

[4] G. Krenn, J.Summhammer, K. Svozil, Phys. Rev. A 61 (2000) 052102

[5] Kwiat, P. G., White, A. G., Mitchell, J. R., Nairz, O., Weihs, G., Weinfurter, H. and Zeilinger, A., preprint available athttp://xxx.lanl.gov/quant-ph/9909083 (1999); A. G. White, J. R. Mitchell, O. Nairz, and P. G. Kwiat, Phys. Rev. A 58, 605 (1998); Tsegaye, T., Goobar, A., Karlsson, A., Bjork, G., Loh, M. Y. and Lim, K. H., Phys. Rev. A 57, 3987-3990 (1998).

[6] W. van Dam, preprint available at http://xxx.lanl.gov/quant-ph/0008059 (2000).

[7] L. K. Grover, Phys. Rev. Lett. 79, 325 (1997)

[8] A. Kent and D. Wallace, quant-ph/0102xxx.

[9] C. W. Helstrom, Quantum Detection and Estimation Theory, (Academic Press, New York,1976)

[10] C. H. Bennett, E. Bernstein, G. Brassard, U. Vazirani, SIAM J. Comput 26 1510-1523 (1997)

[11] E. Fahri and S. Gutmann, Phys. Rev. A 57 (1998) 2403 